\title{
Numerical resistivity calculations for disordered three-dimensional metal models using tight-binding Hamiltonians
}

\author{
Yulia Gilman and Philip B. Allen \\ Department of Physics and Astronomy, SUNY at Stony Brook, Stony Brook, New York 11794-3800, USA \\ Jamil Tahir-Kheli and William A. Goddard III \\ Material and Process Simulation Center, Department of Chemistry, California Institute of Technology, \\ Pasadena, California 91125, USA
}

(Received 26 August 2004; published 1 December 2004)

\begin{abstract}
We calculate the zero-temperature resistivity of model three-dimensional disordered metals described by tight-binding Hamiltonians. Two different mechanisms of disorder are considered: diagonal disorder (random on-site potentials) and off-diagonal disorder (random hopping integrals). The nonequilibrium Green function formalism provides a Landauer-type formula for the conductance of arbitrary mesoscopic systems. We use this formula to calculate the resistance of finite-size disordered samples of different lengths. The resistance averaged over disorder configurations is linear in sample length and resistivity is found from the coefficient of proportionality. Two structures are considered: (1) a simple cubic lattice with one $s$-orbital per site, and (2) a simple cubic lattice with two $d$-orbitals. For small values of the disorder strength, our results agree with those obtained from the Boltzmann equation. Large off-diagonal disorder causes the resistivity to saturate, whereas increasing diagonal disorder causes the resistivity to increase faster than the Boltzmann result. The crossover toward localization starts when the Boltzmann mean free path $l$ relative to the lattice constant $a$ has a value between 0.5 and 2.0 and is strongly model dependent.
\end{abstract}

DOI: 10.1103/PhysRevB.70.224201

PACS number(s): 72.15.Eb, 72.15.Rn, 72.15.Lh

Saturation of resistivity in metallic compounds ${ }^{1}$ as well as its absence ${ }^{2}$ is an interesting phenomenon which is far from fully understood. Some compounds saturate at the levels predicted by the Ioffe-Regel condition ( $l=a$, where $a$ is lattice constant and $l$ is the mean free path), others saturate at much larger levels (i.e., higher resistivity), and there are some that do not saturate at all. This diversity in behavior received substantial attention from theorists. For example, Millis et $a .^{3}$ applied dynamical mean-field theory to calculate resistivity of electrons coupled to phonons and static disorder. Gunnarsson et ll $^{4}{ }^{4}$ studied several rather realistic models with different forms of electron-phonon coupling using a quantum Monte Carlo method. They observed saturation of resistivity in the case of phonons coupled to hopping matrix elements. In an attempt to understand the mechanism of saturation we chose to study transport properties of simple models of metals with static disorder (as opposed to models closely reproducing reality).

The resistivity was calculated using the Landauer-type formula for the zero-temperature linear response of mesoscopic systems, which can be derived in the framework of nonequilibrium Green function formalism. ${ }^{5}$

$$
G=\frac{2 e^{2}}{h} \operatorname{Tr}\left[\Gamma_{L} G^{\mathrm{ret}} \Gamma_{R} G^{\mathrm{adv}}\right]
$$

where $G^{\text {ret }}$ and $G^{\text {adv }}$ are retarded and advanced Green's functions of the system of interest, $\Gamma_{L}$ and $\Gamma_{R}$ are matrices describing the effect of contacts on the system.

The formula above is suitable only for finite samples, whereas our aim is to calculate the resistivity of bulk disordered material as a function of strength of disorder. The so- lution is to calculate resistance of several samples of different lengths and then extract bulk resistivity $\rho$ from the data using the formula $R=L \rho / A$ ( $L$ is length, $A$ is cross section).

Let us describe the setup of calculation for an individual sample. We consider a sample consisting of $N_{x} \times N_{y} \times N_{z}$ unit cells (for our simple cubic examples, each unit cell contains one atom). The sample is placed between two semi-infinite contacts of the same cross section $N_{y} \times N_{z}$. Both contacts and sample have the same crystal structure and are described by a tight-binding Hamiltonian with the same parameters. Then tight-binding parameters for atoms inside the sample are randomly changed from their initial values according to rules given later and the resistance of the disordered sample is calculated.

Our sample is not periodic in the direction of current flow $X$, but, in order to decrease the effect of boundaries on the results, periodic boundary conditions (with period $N_{y}$ and $N_{z}$ ) are used in the perpendicular directions $Y$ and $Z$. Then standard $k$-vector formalism applies in these two directions. For a given $k$-vector, Hamiltonians of the sample and contacts are constructed and the conductance is calculated. Then the conductance is averaged with equal weights over $k$-points on a uniform grid (a $6 \times 6$ grid was used, with a new random Hamiltonian at each $k$-vector).

In the limit of small disorder strength, three-dimensional transport should be accurately described by the linearized Boltzmann equation. Therefore it is useful to compare our numerical results with resistivity obtained from the Boltzmann equation. We did not solve this equation exactly, but used instead the standard procedure of a displaced FermiDirac distribution ${ }^{6} F(k) \sim f_{F D}(k+e E \tau / h)$, where the displacement $e E \tau / h$ to variational accuracy is given by 


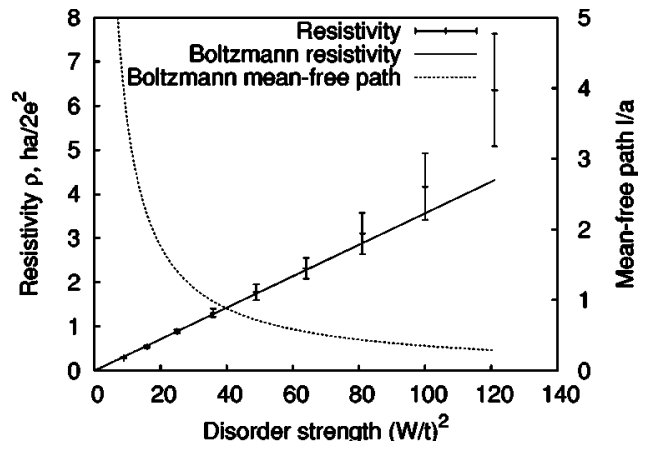

FIG. 1. Resistivity for a single $s$-band model with diagonal disorder. The solid line is the Boltzmann resistivity for the same model. The dashed line shows the Boltzmann mean free path vs $W^{2}$.

$$
\frac{h}{\tau}=2 \pi \frac{\sum_{k k^{\prime}} \overline{\left|V_{k k^{\prime}}\right|^{2}}\left(v_{k}-v_{k^{\prime}}\right)^{2} \delta\left(\epsilon_{k}-\epsilon_{F}\right) \delta\left(\epsilon_{k^{\prime}}-\epsilon_{F}\right)}{2 \sum_{k} v_{k}^{2} \delta\left(\epsilon_{k}-\epsilon_{F}\right)},
$$

where $\hbar v_{k}=\nabla_{k} \epsilon_{k}, V_{k k^{\prime}}$ is the matrix element of scattering potential calculated in Born approximation and the bar indicates the ensemble average.

The first case is a simple cubic crystal, with one atom per unit cell and one $s$-orbital per atom. There are two tightbinding parameters, the energy level $\epsilon_{0}$ (diagonal element of Hamiltonian) which is taken to be zero and the hopping integral $t=1$ between first nearest neighbors (all other hopping integrals are neglected). We consider a half-filled band. Two types of disorder are possible in this model, diagonal and off-diagonal.

In the case of the diagonal disorder, the hopping parameter $t$ is kept constant throughout the sample but the energy level $\epsilon$ is changed randomly according to the formula: $\epsilon=0$ $+\xi$, where $\xi$ is a random variable distributed uniformly in $[-W / 2, W / 2]$. $W$ serves as the measure of disorder strength. The scattering potential $\overline{\left|V_{k k^{\prime}}\right|^{2}}$ is then $W^{2} / 12$.

In this calculation as well as in all others, the cross section of samples is $9 \times 9$, and the lengths used are 5, 6, 7, and 8 . For each length, 36 configurations of disorder are created, the resistance of the sample for each configuration is calculated and then averaged over configurations. This procedure is repeated for different $L$ and $W$. In order to find the bulk resistivity, resistance vs $L$ is plotted for each $W$ and resistivity is found from the slope of the resulting line.

Final results are shown in Fig. 1. The resistivity $\rho_{B}$ calculated using a linearized Boltzmann equation is plotted on the same graph for comparison. The resistivity depends linearly on $W^{2}$ in a quite large range of $W$ (up to $W=8$ ). At larger $W$ it deviates upwards from the Boltzmann resistivity $\rho_{B}$. The dotted curve shows the mean free path $l=\left\langle v^{2}\right\rangle^{1 / 2} \tau$ calculated from Boltzmann resistivity vs $W^{2}$. It can be seen that deviation of resistivity from linear starts approximately when $l / a \sim 0.5$. This result reproduces the earlier work done by Nicolic and Allen ${ }^{7}$ (with a different computer code).

In the case of off-diagonal disorder, the energy level $\epsilon$ is kept constant whereas hopping elements $t$ inside the sample are changed randomly according to $t=1+\xi$, where $\xi$ is again a random variable distributed uniformly in $[-W / 2, W / 2]$.

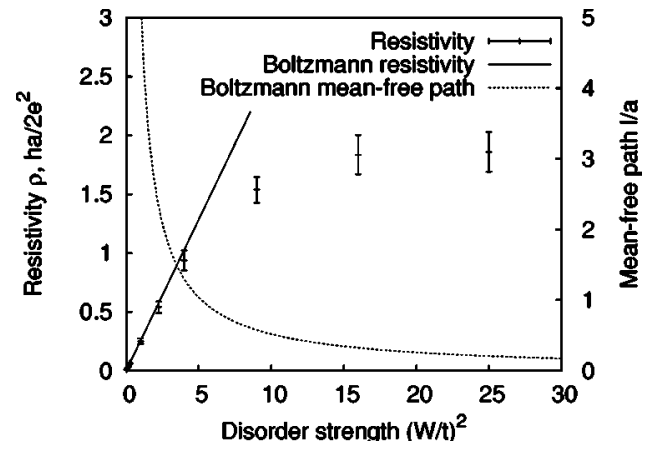

FIG. 2. Resistivity for a single $s$-band model with off-diagonal disorder. The solid line is the Boltzmann resistivity for the same model. The dashed line shows the mean free path vs $W^{2}$.

The calculational procedure is otherwise the same. Results for off-diagonal disorder are shown in Fig. 2. It can be seen that resistivity depends linearly on $W^{2}$ up to $W=2$. At larger $W$, when $l / a \sim 1$, the resistivity deviates from linear, and at $W=4$ it starts to saturate at some level. Therefore in the case of off-diagonal disorder there is no metal-insulator transition (in agreement with the statement of Antoniou and Economou $^{8}$ ). It should also be noted that our result is similar to the results of Calandra and Gunnarsson ${ }^{9}$ obtained for a more realistic model of disorder induced by electron-phonon interaction.

We also studied the combination of diagonal and offdiagonal disorders in order to see how interplay between them would affect resistivity. Diagonal disorder was kept constant and off-diagonal disorder was gradually increased. Some results are shown in Fig. 3. It is clear that the presence of diagonal disorder can change the resistivity vs offdiagonal disorder dependence. At the diagonal disorder $W_{\text {diag }}=11$ resistivity decreases with increasing off-diagonal disorder. At $W_{\text {diag }}=6$ resistivity displays nonmonotonic behavior. In all cases resistivity saturates eventually at approximately the same level as in the absence of static diagonal disorder (see Fig 2).

Next we consider a simple cubic structure with one atom per unit cell, but two $d$-orbitals per atom, namely $\left|x^{2}-y^{2}\right\rangle$ and $\left|3 z^{2}-r^{2}\right\rangle$ ( $E_{g}$ orbitals). As in the previous model, only first nearest neighbor hopping is considered. In this case, all hopping elements can be expressed in terms of two coupling

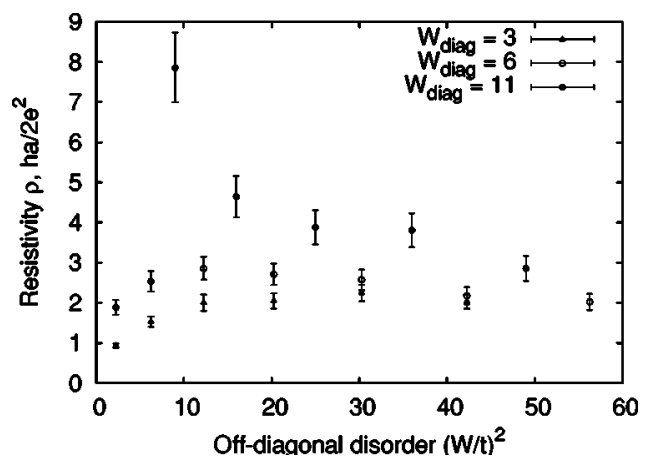

FIG. 3. Resistivity for a single $s$-band model with a combination of disorders. Diagonal disorder is kept constant, off-diagonal disorder varies. 


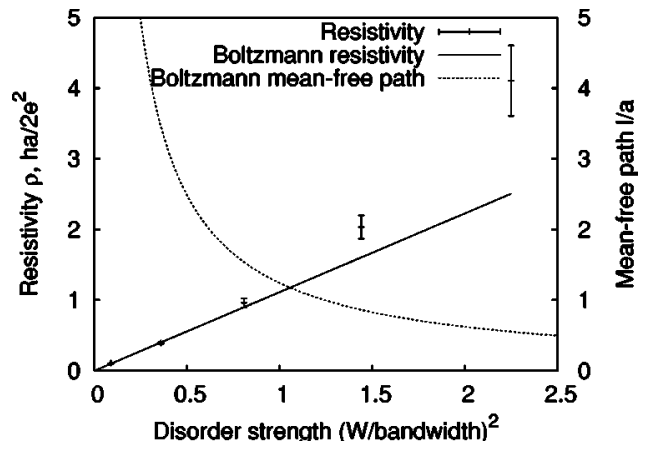

FIG. 4. Resistivity for the $E_{g}$ model with diagonal disorder. The solid line is the Boltzmann resistivity for the same model. The dashed line shows the mean free path vs $W^{2}$. It can be seen that resistivity deviates from the Boltzmann expression when $l$ becomes close to lattice constant $a$.

parameters $(d d \sigma)$ and $(d d \delta)$ which are taken to be 0.051 and $0.003 \mathrm{eV}$, respectively. Both orbitals have energy levels set to zero.

First, diagonal disorder with a uniform distribution was considered. The two $E_{g}$ orbitals are given independent random diagonal energies. Disorder strength $W$ is measured in terms of the full bandwidth, which for the present choice of parameters equals $0.3 \mathrm{eV}$. The resulting graph of resistivity vs $W^{2}$ is shown in Fig. 4 along with the Boltzmann resistivity. As in the case of a single orbital model, the resistivity is initially linear in $W^{2}$ and then begins to deviate upwards when $l / a \sim 1$, indicating the presence of a metal-insulator transition at larger $W$.

Off-diagonal disorder in the case of two $d$-orbital models can be created in numerous ways since there are several different hopping elements in the Hamiltonian. We chose disorder strength to be proportional to the hopping element itself, or more specifically: $t-t_{0}=t_{0} * \xi$, where $\xi$ is defined above. Results are shown in Fig. 5 and are similar to those of the

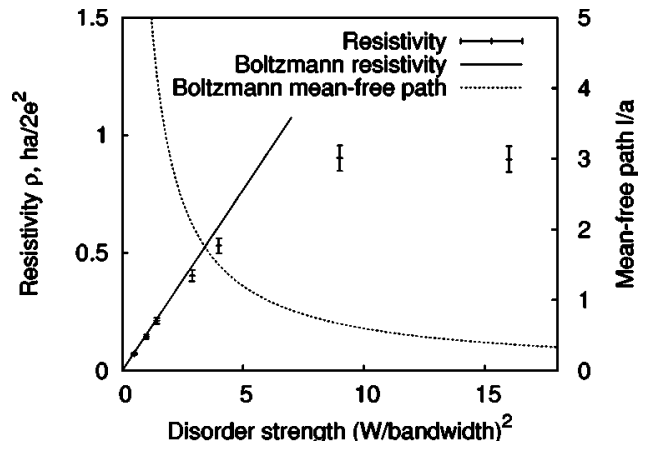

FIG. 5. Resistivity for the $E_{g}$ model with off-diagonal disorder. The solid line is the Boltzmann resistivity for the same model. The dashed line shows the mean free path vs $W^{2}$. It can be seen that resistivity deviates from Boltzmann expression when $l$ becomes close to lattice constant $a$.

single $s$-orbital model. The resistivity starts to deviate from Boltzmann-like when $l / a \sim 2$, and to saturate when $l$ comes close to the lattice constant, in agreement with Ioffe-Regel condition.

The main conclusion to be drawn is that the key factor to resistivity saturation in metals is strong off-diagonal disorder. The combination of diagonal and off-diagonal disorder can produce various types of the resistivity behavior: decreasing, increasing, or nonmonotonic. Saturation is found even in the case of a single band, which is in disagreement with the statement of Allen and Chakraborty ${ }^{10}$ that multiband structure is essential for saturation. The value of $l / a$ at which Boltzmann theory starts to break down varies surprisingly strongly, from 0.5 to 2.0 , for the four models considered here.

Financial support for Y.G. and P.B.A. was provided by NSF (DMR-0089492). Financial support for J.T.K. and W.A.G. was provided by MARCO-FENA and by NSF (DMR-0120967).
${ }^{1}$ Z. Fisk and G. W. Webb, Phys. Rev. Lett. 36, 1084 (1976).

${ }^{2}$ M. Gurvitch and A. T. Fiory, Phys. Rev. Lett. 59, 1337 (1987); A. F. Hebard T. T. M. Palstra, R. C. Haddon, and R. M. Fleming, Phys. Rev. B 48, 9945(R) (1993).

${ }^{3}$ A. J. Millis, J. Hu, and S. Das Sarma, Phys. Rev. Lett. 82, 2354 (1999).

${ }^{4}$ M. Calandra and O. Gunnarsson, Phys. Rev. B 66, 205105 (2002); M. Calandra and O. Gunnarsson, Europhys. Lett. 61, 88 (2003); O. Gunnarsson and J. E. Han, Nature (London) 405, 1027 (2000).

${ }^{5}$ Y. Meir and N. S. Wingreen, Phys. Rev. Lett. 68, 2512 (1992).
${ }^{6}$ P. B. Allen, in Quantum Theory of Real Materials, edited by J. R. Chelikowsky and S. G. Louie (Kluwer, Boston, 1996), Chap. 17, p. 219.

${ }^{7}$ B. K. Nikolic and P. B. Allen, Phys. Rev. B 63, 020201(R) (2001).

${ }^{8}$ P. D. Antoniou and E. N. Economou, Phys. Rev. B 16, 3768 (1977).

${ }^{9}$ O. Gunnarsson, M. Calandra, and J. E. Han, Rev. Mod. Phys. 75, 1085 (2003).

${ }^{10}$ P. B. Allen and B. Chakraborty, Phys. Rev. B 23, 4815 (1981). 